\title{
An Approach for Creating Business Model for Using Virtual Tools in Startup Companies
}

\author{
Assoc. Prof. Dr. Pavel Petrov \\ University of Economics - Varna, Varna, Bulgaria \\ petrov@ue-varna.bg \\ $\mathrm{PhD}$ candidate Svetoslav Ivanov \\ University of Economics - Varna, Varna, Bulgaria \\ svetoslav_ivanov@ue-varna.bg \\ PhD candidate Volodymyr Petrivskyi \\ Taras Shevchenko National University of Kyiv, Kyiv, Ukraine \\ vovapetrivskyi@gmail.com
}

\begin{abstract}
The forms of business organizations follow some steady trends driven by tendencies like the rapid development of IT and the transition of information systems. The application of virtualization and the use of virtual tools by start-up companies are discussed. The article reviews the phases of the business model and software product development. The activities in them are grouped and sample tools for each is listed. Also, the modern form of organization as the virtual office and corporation are reviewed as becoming necessary. In detail, the startup company is reviewed in the form of a virtual corporation with its characteristics. They are classified and areas of application and management issues are discussed. Finally, the coverage of activities with virtual tools is evaluated and summarized.
\end{abstract}

Keywords: business organization, startup, virtualization, virtual corporation

JEL Code: O33 DOI: https://doi.org/10.36997/IJUSV-ESS/2020.9.3.19

\section{Introduction}

Over the last two decades, there have been steady trends worldwide that have strongly influenced the forms of business organization.

First, there is an increase in custom production, which is associated with increasing market segmentation. This requires the application of new marketing and production concepts, which leads to changes in the organization of business in the direction of greater flexibility and increased synchronization with business partners.

Second, there is a strong and rapid development of information and communication technologies, which leads to increased demand for IT professionals to maintain and develop the traditional information infrastructure and information system of a business organization. As there is a shortage in the labour market of skilled IT professionals, much of the activity can be outsourced to reduce IT costs (Petrov et al., 2019; Petrov et al., 2020). For the needs of the management of the joint activity, it is necessary to use a common virtual information system (Iliev et al., 2010).

Third, globalization is intensifying as a result of political changes that have engulfed much of the world. This enables business organizations with smaller sizes to enter new territories and markets, for this purpose territorially remote structures are created, most often in the form of corporations, to perform specific tasks of entering new markets and realization of new projects.

Fourth, again due to globalization, the business environment of a certain existing market segment is changing. The easy entry of new "external" competitors leads to increased competition in the specific market segment. To protect their positions, existing business organizations need to optimize their activities, and by reducing operating costs and creating a new type of more flexible organizational structures, they could counteract their competitors more successfully.

The above factors are among the main prerequisites for the effective implementation of a virtual work organization in one or more business organizations and the formation of virtual 
corporations.

\section{Business model in software development}

The main task in the formation of a technology company is to create a business model and its market validation. The business model contains a concept for the product named with different terms, such as product, unique value proposition, minimum functioning product (MVP) (Alvarez, 2014; Maurya, 2012; Ries, 2011).

Validation of the model is done regularly to a specific market by potential or actual customers. Validity tests are performed, data are collected from them for the analysis, knowledge extraction, and decision-making for correction in the model and technological solution. Therefore, the selected indicators must be directly related to the activity and lead directly to decision-making. Different systems of indicators are described in the literature for different business models (Adler, 2011; Blank, 2013):

- AARRR to measure the necessary actions to Dave McClair's clients;

- Engines of Growth by Eric Rees;

- Easy start scheme (Lean Canvas) of Ash Moria;

- Sean Ellis' Growth Pyramide;

- Sales Funnel (Long Funnel) - the transition between stages in the sales process;

- Croll and Yoskowitz Lean Analytics Framework.

Their choice and use depend on the process. The full testing of a business model is evaluated regularly. Tools for this stage are also available. Exemplary ones are listed in the Table. 1.

Table 1. Activities and virtual tools for the "Business Model" phase.

\begin{tabular}{|l|l|}
\hline \multicolumn{1}{|c|}{ Activity } & \multicolumn{1}{c|}{ Tool } \\
\hline Creating & $\begin{array}{l}\text { Model research, (un)successful experiments, indicators: leanstack.com, } \\
\text { leananalyticsbook.com, startups.co, bizplan.com; } \\
\text { Advice from mentors: clarity.fm, fi.co, professional social networks and means of } \\
\text { communication; } \\
\text { Virtual office with description documents; } \\
\text { MVP through a list of requirements or a prototype; }\end{array}$ \\
\hline Testing & $\begin{array}{l}\text { Creating database clients: mailchimp.com, launchrock.com, } \\
\text { Web traffic analysis: indicative.com, manageengine.com, } \\
\text { analytics.google.com, analytics.angelfishstats.com/, adobe.com/analytics/adobe- } \\
\text { analytics.html; } \\
\text { *arketing Behavior Analysis: clicky.com, kissmetrics.com, shopmetrics.com, } \\
\text { the business model, the } \\
\text { evaluation approach, } \\
\text { indicators, and tools. } \\
\text { The data are indicative. } \\
\text { retentiongrid.com, adobe.com/marketing-cloud.html, metrilo.com, } \\
\text { marketingplatform.google.com; } \\
\text { Polls: docs.google.com/forms, jotform.com, startupstash.com/forms-surveys, } \\
\text { marketingplatform.google.com; } \\
\text { Tests: adobe.io/apis/experiencecloud/target.html, abtasty.com/, optimizely.com, }\end{array}$ \\
\hline $\begin{array}{l}\text { Extraction of } \\
\text { knowledge }\end{array}$ & $\begin{array}{l}\text { Test data processing with standard analysis: docs.google.com/spreadsheets, } \\
\text { tableau.com/products/cloud-bi, sas.com/en_us/solutions/cloud-computing.html, } \\
\text { turbo.net/hub/powerbi (Power BI online), sisense.com; }\end{array}$ \\
\hline Correction & not specified \\
\hline Presentation & presentations and diagrams \\
\hline
\end{tabular}

It can be noted that there are a variety of virtual tools and data sources for this phase. No tools are specified for the "Correction" activity because it is the result of analyzing results and making decisions for change.

The activities in the phase "Business Model" and the next phase "Software Development" are performed step by step with a flexible methodology, sequentially or in parallel at the choice of the entrepreneur. The choice depends on the degree of need for a prototype, real or simulated 
fulfillment of minimum performance requirements (MVP) over time. Regarding software development, the tools used depend on the chosen business model, software architecture, and implementation technology. Typically, the product is realized through a multi-layer architectural solution with separate interconnected layers, and each layer has multiple components. There are single platforms for single-vendor development and complementarity with third-party components that are missing or more efficient than standard platforms. Therefore, virtual tools can be platformrelated or widely applicable.

Table 2 presents the standard basic and ancillary software development activities described in the literature (Poppendieck, 2003; Schwaber \& Beedle, 2001).

Table 2. Activities and virtual tools for the "Software Development" phase.

\begin{tabular}{|c|c|}
\hline Activity & Tool \\
\hline Analysis & $\begin{array}{l}\text { Virtual office for documents } \\
\text { Design tools (Design activity) } \\
\text { Description of requirements: atlassian.com/software/confluence }\end{array}$ \\
\hline Design & $\begin{array}{l}\text { Modeling tools: lucidchart.com, cacoo.com, } \\
\text { sparxsystems.com/products/procloudserver/, dragon1.com/products/dragon1-connect- } \\
\text { edition, products.office.com/en-us/Visio/, axure.com, figma.com }\end{array}$ \\
\hline $\begin{array}{l}\text { Realization } \\
* \text { Note: many } \\
\text { environments support } \\
\text { the integration of } \\
\text { other activities and a } \\
\text { wide range of } \\
\text { products. }\end{array}$ & $\begin{array}{l}\text { Coding: codeanywhere.com, aws.amazon.com/cloud9, koding.com, } \\
\text { progress.com/kinvey, azure.microsoft.com/en-us/products/visual-studio-code, } \\
\text { aws.amazon.com / lambda, telerik.com/app-development; } \\
\text { Databases: azure.microsoft.com/en-us/services/sql-database/, azure.microsoft.com/en- } \\
\text { us/product-categories/databases/, aws.amazon.com/products/databases; } \\
\text { Business logic: aws.amazon.com/lambda, azure.microsoft.com/en-us/product- } \\
\text { categories/compute/; } \\
\text { Web server: azure.microsoft.com/en-us/product-categories/web, } \\
\text { Cloud services: Providers have many services - from API, computing, balancing, } \\
\text { queuing to artificial intelligence; } \\
\text { Module repositories: nuget.org, npmjs.com, github.com, openwrap.org, } \\
\text { incubator.apache.org/npanday,chocolatey.org; } \\
\text { User interface: angular.io, reactjs.org, vuejs.org, startbootstrap.com, mockplus.com, } \\
\text { jqueryui.com, jqwidgets.com, riot.js.org, semantic-ui.com, webix.com; }\end{array}$ \\
\hline $\begin{array}{l}\text { Testing - } \\
\text { Verification and } \\
\text { Validation }\end{array}$ & $\begin{array}{l}\text { Automated integration and installation: github.com, jenkins.io, gitlab.com, } \\
\text { circleci.com, cloudbees.com, azure.microsoft.com/en-us/services/devops/; } \\
\text { Test systems: gurock.com/testrail, seleniumhq.org, getxray.app, } \\
\text { techexcel.com/products/devtest/, ranorex.com, getzephyr.com; } \\
\text { Automated tests: ranorex.com, https://percy.io/, Associate testers: utest.com/about-us, }\end{array}$ \\
\hline Implementation & $\begin{array}{l}\text { Automated installation tools: jenkins.io, azure.microsoft.com, www.gitlab.com, } \\
\text { cloudbees.com,; }\end{array}$ \\
\hline Support & atlassian.com/software/jira/service-desk, atlassian.com/software/jira/ops, \\
\hline $\begin{array}{l}\text { Project } \\
\text { management }\end{array}$ & $\begin{array}{l}\text { azure.microsoft.com/en-us/services/devops, } \\
\text { atlassian.com/software/jira, atlassian.com/software/trello, } \\
\text { github.com/features/project-management, gitlab.com, }\end{array}$ \\
\hline $\begin{array}{l}\text { Configuration and } \\
\text { change management }\end{array}$ & gitlab.com, atlassian.com/software/crucible, \\
\hline $\begin{array}{l}\text { Configuring the } \\
\text { work environment }\end{array}$ & $\begin{array}{l}\text { Tool selection and setup: azure.microsoft.com, } \\
\text { Programming, project management environments (above); } \\
\text { Repository: github.com, atlassian.com/software/bitbucket }\end{array}$ \\
\hline
\end{tabular}

Sample virtual tools for developing a multi-layered web solution are included, mainly with technologies and products related to the development platform of Microsoft and Amazon. The results show that there is a wide variety of suppliers, as well as coverage of activities. Integrations between tools are popular in practice. We should note the three models of integration between tools: 
- The main product is desktop, but integrates with many virtual tools (Example Microsoft Visual Studio with packages and cloud services);

- Embedded integration through an event from another service/instrument;

- Integration of tools through additionally privately developed event processing.

\section{Technology company in the form of a virtual corporation.}

A virtual corporation is a business organization consisting of geographically remote independent legal entities or individuals - members: business partners and employees. They interact with each other mainly by electronic means of communication to perform a common task or common business. The virtual corporation can be legally differentiated as a legal entity or the participants in the virtual corporation can consider it as such, without being advertised as an independent legal entity. In the latter case, the accounting can be performed by each of the business partners individually or by one of them by agreement.

In case the virtual corporation is not legally differentiated as a corporation, it does not have its separate property and the property belongs to the individual business partners. This allows you to dynamically reallocate resources between different tasks and respond in a minimum of time depending on the situation (Petrov\&Valov, 2019). In private cases, it is even possible that the information system used does not belong to any of the members - it is free software, which in most cases is free and is installed on a server that provides free hosting services, and for IT infrastructure to use public communications. network.

The main characteristics of the virtual corporation are the following: its members are geographically remote, communication is carried out through modern information and communication technologies (Panayotova et al., 2016; Dimitrov et al., 2020a; Dimitrov et al., 2020b), intangible activity predominates, specific products, and services are created. Very often the members of the virtual corporation are prosumators, i.e. they use the finished product by themselves.

The division of the activity into separate parts - for a specific member, increases the division of labour, and hence the productivity of labour, as there is a possibility to change or duplicate the work performed by some of the members. A virtual corporation can be defined as successful if with lower costs, shorter time, or higher quality it copes with a certain task, compared to another organizational form. Ideally, in the virtual corporation, there is a synergistic effect, which leads to increased efficiency of each participant and the corporation as a whole.

In a broad sense, virtuality is defined as something that does not exist in reality - something simulated, imaginary (Kostadinova et al., 2018). The term "virtual corporation" should be understood in a narrow specific rather than a broad sense, as it is completely virtual, i.e. without the presence of people and a formal logical structure in the real world, a corporation cannot exist. In this context, the word "virtual" means that in the corporation real people intensively use modern information and communication technologies to interact with each other. These technologies are used to increase the level of integration and coordination between the members of the corporation. Each of the members of a virtual corporation usually has a unique resource that he shares with others and that supports the activity. These resources can be intellectual capabilities (in software outsourcing, design, translation services), access to end customers (in tourism, real estate, marketing research, outsourcing, etc.), availability of means of production or equipment (printing machine, CNC machines), etc. In turn, each resource can be used in several virtual corporations at different times and so the unique resources can be shared dynamically, depending on the current needs of different business organizations. Therefore, some researchers emphasize that the virtual corporation is temporary, but in our opinion, this temporary nature is relative to the time of existence of each resource taken separately, because, with a lasting effect of the cooperation, the virtual corporation can last long, unlimited in duration, and periods of time. 
The main factor that facilitates the emergence of a virtual corporation is the Internet, which allows virtual corporations to operate efficiently, with low communication costs. An additional factor is a need for companies to use the services of highly qualified employees, experts in their field, without the need to relocate them, which in most cases can mean migrating to a foreign country. The development of the network infrastructure and especially of the so-called "last kilometre" - where the connection between the end customer and the Internet provider takes place, the development of grouper systems, the availability of cheap options for audio and video conferencing, the dynamics of the labour market are also important factors that determine the emergence and the development of the virtual corporation.

In a virtual corporation, the activity of employees may not be performed in one country, city, or territory, and the physical territorial division of employees allows optimal selection of a team for work, as in each region there are potential employees with different qualifications and different payment standard. Due to the peculiarities of this form of organization, the information technologies used are of high importance for the success of the virtual corporation. They affect both the speed with which the process of information exchange between members takes place and the ability of the corporation to make the most of the synergistic effect, regardless of the asynchronous distribution of activities over time caused by distances (members may be in different time zones). The way e-mail, grouper, and the Internet's ability to organize audio and video conferencing let the virtual corporation be more flexible, faster, and generally more efficient.

Some authors divide virtual corporations into two types - virtual enterprise and virtual office, when it comes to remote workers from different companies that produce a common product and remote employees of a company, interacting with each other. They interact mainly by electronic means of communication and using a common system for the exchange, storage, processing, and transmission of data and control actions (Malecki\&Moriset, 2008). This division corresponds to the division of intra-company and inter-company associations proposed by other authors: "The virtual organization of business manifests itself in two forms: intra-company network associations and inter-company network associations, the former involving company employees and the latter partners."

From the point of view of the lifespan of virtual corporations, they can be classified into four categories:

- static virtual office (intra-company / intra-company static virtual corporation) - long-term virtual organization between employees of a company, to improve work efficiency;

- dynamic virtual office (in-house dynamic virtual corporation) - a temporary virtual organization between employees in different branches/departments to work on a specific project;

- static virtual enterprise (intercompany static virtual corporation) - a leading company provides part of the ancillary activities to subcontractors - specialized companies, based on longterm contracts (e.g. accounting, hiring, software and computer network maintenance, warranty service, etc. n.);

- a dynamic virtual enterprise (inter-firm dynamic virtual corporation) - a short-term virtual association with a precisely defined and achievable goal - most often to respond quickly to a specific market need.

The main components of a virtual corporation are the virtual workplace and the virtual information system (e.g. a web-based information system) through which the employee works. Most often, the connection between these two components is made through the World Wide Web, due to the low cost of communication and the possibility of territorial independence of both the employee and the virtual information system.

The workplace is a computer (usually portable) with software installed to connect and work in the remote virtual information system. The physical connection of the workplace to the system can be organized in different ways - via modem, LAN-card, GPRS, wireless connection, etc., which allows the workplace to be located in different parts of a large area where there is Internet access. 
(e.g. the territory of the European Union, the United States, etc.) at relatively low relocation costs. The lack of stationarity is in some situations one of the biggest advantages of the virtual workplace compared to the classic version.

The virtual information system that supports the work of virtual workstations must include server applications that implement various functions used by the remote employee.

The opportunities for the employee to work from the virtual workplace are in two main directions:

- access to the necessary corporate information, and depending on its functions the employee can read, write or change data stored on the server part;

- to communicate and exchange data with other employees of the virtual corporation.

Suitable areas of application of the virtual organization are software development and maintenance, real estate sales, booking activities in tourism, as well as activities that are accompanied by constant relocation and work in unusual places.

Regarding the location of the resources of the virtual corporation, we can distinguish them as centralized - the main resources are located in one place and decentralized - the resources are distributed approximately evenly among the members. The latter option is most common when members of a virtual corporation have their external business relationships and are relatively more independent in their work.

The management style of the virtual corporation can change depending on the task and the degree of its implementation. For example, decentralized informal management is most common in the development of free and open-source software - the so-called "market" style, which in this case some authors consider more successful than the traditional hierarchical-centralized "cathedral" management style. . In decentralized management, usually each of the participants benefits from the result of the joint work (prosumator).

In the centralized management model, one of the members has a leading role and distributes the tasks, and determines the functions of the other members. There is a similar structure in software development in the so-called. outsourcing. A leading software company that manages financial resources assigns tasks to other members - for example, interface development, database design, coding, testing, and more. In case of failure to perform the tasks, one of the members of the virtual corporation can be replaced or the work can be duplicated by another member (such duplication is appropriate in case compliance with the deadline is very important), and the decision is made by the lead participant. which in turn is irreplaceable.

\section{Coverage of activities with virtual tools}

In summary of what has been presented so far, for almost every activity there are virtual tools available as services. It is up to the entrepreneur to decide which of them and how convenient they are for a given product, idea, and business model. In terms of price, most tools have zero or low price - free versions, free for micro teams (up to 5-10 users) or the price is according to the number of users or volume of consumption. This is a convenience for a start-up company, as it implies low operating costs. A significant problem could be a variety of tools to use. In contrast, many products have features that cover more than one activity and/or can be integrated with thirdparty tools for other activities, which makes it easier to build an integrated startup environment.

Following the results presented in this way, we can make a summary of the extent to which activities in the entrepreneurial process can be performed with virtual tools. We should take this into account with two indicators - coverage and diversity. The first indicator will answer the question of how many virtual tools are available for the tasks in the activity. The second, diversity, is the degree of possible choice of various available tools. For their presentation, we will use a relative scale with numbers from 0 to 3 , which is given in table. 3 as a synthesis of the two indicators. It does not reflect a ratio, but rather a grouping by these two indicators in order to more clearly reflect and present the degree of coverage of tasks by activities. 
Table 3 . The scale of activity coverage with virtual tools.

\begin{tabular}{|c|c|c|c|}
\cline { 2 - 4 } \multicolumn{1}{c|}{} & \multicolumn{3}{c|}{ Diversity } \\
\hline Coverage & Low & Medium & High \\
\hline None & 0 & 0 & 0 \\
\hline Low & 1 & 1 & 2 \\
\hline Average & 1 & 2 & 3 \\
\hline High / Full & 2 & 3 & 3 \\
\hline
\end{tabular}

Following the given indicators and the given scale, we can summarize the results of the presented tools in Table 4.

Table 4. Coverage of activities by phases with virtual tools.

\begin{tabular}{|c|c|c|c|c|}
\hline Preparation & Invention & Business model & \multicolumn{2}{|c|}{ Software development } \\
\hline Suitability & Study & Creating & Analysis & The project \\
\hline 2 & 2 & 2 & 2 & 2 \\
\hline Training & Issue & Testing & Design & Configurations \\
\hline 2 & 1 & 3 & 2 & 2 \\
\hline Communication & Idea & Knowledge & Realization & Environment \\
\hline 3 & 1 & 2 & 3 & 3 \\
\hline Virtual Office & Strategic Analysis & Correction & Testing & Implementation \\
\hline 3 & 1 & 0 & 3 & 2 \\
\hline Environment & Idea selection & Presentation & Validation & Support \\
\hline 1 & 0 & 2 & 2 & 2 \\
\hline
\end{tabular}

In Table 4 we list 25 activities. Activity "Presentation of an idea" and "Presentation of a business model" are combined due to the coverage of the tools. As can be seen from Table 4, for 2 activities there are no specified tools and 4 are low, 13 are medium and 6 are high coverage and variety. Approximately $75 \%$ of the activities have available virtual tools with medium or high coverage and variety. This gives grounds to confirm to a significant extent the hypothesis that a significant part of the tasks for a new product in a start-up software company can be performed with virtual tools. The lowest coverage is in the "Invention" phase and the highest in "Software Development". There are opportunities for innovation in the tools for start-ups in two directions increasing the coverage of tasks by activities and integration in a single environment.

\section{Conclusion}

The forms of business organizations follow some steady trends. Custom production, the development of IT, and the transition of information systems, globalization, and easy entry of new competitors are the main prerequisites for virtual work and virtual corporations. The application of virtualization is illustrated with the use of virtual tools by start-up companies. The article reviews the phases of the business model and software product development. Their activities are grouped and sample tools for each one are listed, as well as models of integration between the tools. The 
modern form of organization as the virtual work and corporation has already become necessary in many areas and brings significant benefits to start-up companies. The form of a virtual corporation with its characteristics is reviewed, including resource sharing, facilities, Internet, performance, and lifespan. The virtual organizational forms are classified into four groups. Areas of application, as well as the location of resources and management issues, are discussed. Finally, the coverage of activities with virtual tools is evaluated and summarized. Approximately $75 \%$ of the activities have available virtual tools with medium or high coverage and variety.

\section{References}

1. Adler, C. (2011). Ideas are overrated: startup guru Eric Ries radical new theory. Wired.

2. Alvarez, C. (2014). Lean Customer Development: Building Products Your Customers Will Buy. O'Reilly Media, Inc.

3. Blank, S. (2013). The Four Steps to the Epiphany. K\&S Ranch Publishing.

4. Dimitrov, G., Bychkov, O., et al. (2020). Creation of Biometric System of Identification by Facial Image. In 2020 3rd International Colloquium on Intelligent Grid Metrology (SMAGRIMET): $\quad$ IEEE. pp.29-34. DOI: https://doi.org/10.23919/SMAGRIMET48809.2020.9263995

5. Dimitrov, G., et al. (2020). Increasing the Classification Accuracy of EEG based Braincomputer Interface Signals. In 2020 10th International Conference on Advanced Computer Information Technologies (ACIT): IEEE, pp.386-390. DOI: https://doi.org/10.1109/ACIT49673.2020.9208944

6. Iliev, P., Salov, V., \& Petrov, P. (2010). Virtualni sistemi. Varna: Nauka i ikonomika,

7. Kostadinova, I., Toshev, R., et al. (2018). Temporal Analysis of the Pedagogical Adoptions use and Application of the Augmented and Virtual Reality Technologies in Technical Subject Areas. In 11th Annual International Conference of Education, Research and Innovation, ICERI2018 Proceedings, Seville, Spain: IATED, pp.4387-4393.

8. Malecki E., \& Moriset B. (2008). The Digital Economy. London \& New York, Routledge, p.77. [Online] Available from: http://books.google.com/books?id=MNuXSvs_MkEC [Accessed 10/10/2020]

9. Maurya, A. (2012). Running lean: iterate from plan A to a plan that works. The lean series (2nd ed.). Sebastopol, CA: O'Reilly.

10. Panayotova, G., Dimitrov, G., et al. (2016) Modeling and data processing of information systems. In 3rd International Conference on Artificial Intelligence and Pattern Recognition (AIPR), Lodz, Poland: IEEE, pp.154-158.

11. Petrov, P., \& Valov, N. (2019). Strategic and Tactical Problems in Fintech and E-business Companies. Izvestia Journal of the Union of Scientists - Varna. Economic Sciences Series, 8(3), pp.55-61. DOI: https://doi.org/10.36997/IJUSV-ESS/2019.8.3.55

12. Petrov, P., Buevich, A., Dimitrov, G., et al. (2019). A Comparative Study on Web Security Technologies Used in Bulgarian and Serbian Banks. In 19 International Multidisciplinary Scientific Geoconference SGEM 2019: Conference Proceedings, 19(2.1), pp.3-10.

13. Petrov, P., Ivanov, S., et al. (2020). Opportunities to Use Virtual Tools in Start-Up Fintech Companies. In 20th International Multidisciplinary Scientific Geoconference - SGEM 2020, 20(2.1), pp.247-254. DOI: https://doi.org/10.5593/sgem2020/2.1/s07.032

14. Poppendieck, M., \& Poppendieck, T. (2003). Lean Software Development: An Agile Toolkit. Addison-Wesley.

15. Ries, E. (2011). The Lean Startup. Crown Publishing Group.

16. Schwaber, K., \& Beedle, M. (2001). Agile Software Development with Scrum. Upper Saddle River, NJ: Prentice Hall. 